\title{
BOUNDARY VALUES OF ANALYTIC SEMIGROUPS AND ASSOCIATED NORM ESTIMATES
}

\author{
ISABELLE CHALENDAR \\ Université de Lyon; Université Lyon 1; INSA de Lyon; Ecole Centrale de Lyon \\ CNRS, UMR5208, Institut Camille Jordan; \\ 43 bld. du 11 novembre 1918, F-69622 Villeurbanne Cedex, France \\ E-mail: chalenda@math.univ-lyon1.fr \\ JEAN ESTERLE \\ IMB, Université Bordeaux 1 \\ 351 cours de la Libération, 33405 Talence Cedex, France \\ E-mail: jean.esterle@math.u-bordeaux1.fr \\ JONATHAN R. PARTINGTON \\ School of Mathematics, University of Leeds \\ Leeds LS2 9JT, U.K. \\ E-mail: j.r.partington@leeds.ac.uk
}

\begin{abstract}
The theory of quasimultipliers in Banach algebras is developed in order to provide a mechanism for defining the boundary values of analytic semigroups on a sector in the complex plane. Then, some methods are presented for deriving lower estimates for operators defined in terms of quasinilpotent semigroups using techniques from the theory of complex analysis.
\end{abstract}

1. Introduction. We are interested here in the behaviour near the origin of semigroups which are analytic in a sector $\mathcal{S}_{\alpha}=\{z \in \mathbb{C} \backslash\{0\}:|\arg (z)|<\alpha\}$, and we wish to define in some natural sense their "boundary values" on the boundary $\partial \mathcal{S}_{\alpha}$ of $\mathcal{S}_{\alpha}$. There is an extensive literature concerning strongly continuous semigroups $(T(t))_{t>0}$ of bounded operators on a Banach space, and all the results of the present paper can be formulated in this framework. We made the choice here to adopt the point of view of Banach algebras, and consider analytic semigroups in Banach algebras, i.e., analytic maps $t \mapsto T(t)$ from $\mathcal{S}_{\alpha}$ into a Banach algebra $\mathcal{A}$. We will always assume that the subalgebra generated by

2010 Mathematics Subject Classification: Primary 47D03, 46J40; Secondary 46H20.

Key words and phrases: analytic semigroups, quasimultipliers, sectorial semigroups. The paper is in final form and no version of it will be published elsewhere. 
the semigroup is dense in $\mathcal{A}$, and $\mathcal{A}$ is not unital if the generator of the semigroup is unbounded.

Thus, in Section 2, we consider the algebra $Q M(\mathcal{A})$ of quasimultipliers and the algebra $Q M_{r}(\mathcal{A})$ of regular quasimultipliers on $\mathcal{A}$ defined by the second author in $[9$. This general theory is applied to analytic semigroups in Section 3. In the general case the semigroup $T(t)$ extends to a group of quasimultipliers on $\mathcal{A}$, and the semigroup $\left(T(t)_{t \in \overline{\mathcal{S}_{\alpha}}}\right.$ can be considered as a strongly continuous semigroup of bounded multipliers on a dense ideal of $\mathcal{A}$ which is a Fréchet $\mathcal{A}$-module. When the semigroup admits exponential growth on the lines $\left\{\delta+t e^{i \alpha}\right\}_{t \geq 0}$ and $\left\{\delta+t e^{-i \alpha}\right\}_{t \geq 0}$ for some $\delta>0$, we have $(T(t))_{t \in \overline{\mathcal{S}_{\alpha}}} \subset Q M_{r}(\mathcal{A})$, and $(T(t))_{t \in \overline{\mathcal{S}_{\alpha}}}$ can be considered as a strongly continuous semigroup of multipliers on a dense ideal $I$ of $\mathcal{A}$ which is a Banach $\mathcal{A}$-module. So for this class of semigroups there exists a norm decreasing monomorphism $\theta$ from $\mathcal{A}$ into a Banach algebra $\mathcal{B}$ with dense range such that $\sup _{0 \leq|t| \leq 1, t \in \mathcal{S}_{\alpha}}\|\theta(T(t))\|<+\infty$, and the semigroup $\left(\theta(T(t))_{t \in \mathcal{S}_{\alpha}}\right.$ admits an extension to the multiplier algebra $M(\mathcal{B})$ of $\mathcal{B}$ which is strongly continuous on $\mathcal{B}$. Moreover the generator of $\left(\theta(T(t))_{t \in \mathcal{S}_{\alpha}}\right.$ is unbounded if the generator of the given semigroup is unbounded.

In Section 4 we use these ideas to obtain lower estimates for the distance between elements of the given semigroup. Thus, using the classical Ahlfors-Heins theorem [5, pp. 115-116], we apply these results to show that if $(T(t))_{t \in \mathbb{C}^{+}}$is a quasinilpotent semigroup on the open half plane, and if there exists $\delta>0, \mu>0$ and $\lambda>0$ such that $\sup _{n \in \mathbb{Z}} \lambda^{|n|}\|T(\delta+i n \mu)\|<+\infty$, then we have, for every $y \geq 0$ and every $h>0$,

$$
\max _{\nu= \pm 1} \liminf _{\epsilon \rightarrow 0^{+}}\|T(i \nu y+\epsilon)-T(i \nu(y+h)+\epsilon)\| \geq 2 .
$$

Some very general results for the non-quasinilpotent case may be found in [3].

We conclude this section with a review of the general theory of Fréchet spaces and their operators. Let $V$ be a (complex) linear space equipped with a nondecreasing sequence $\left(\|\cdot\|_{n}\right)_{n \geq 1}$ of seminorms, with $\bigcap_{n \geq 1}$ ker $\|\cdot\|_{n}=\{0\}$. The topology associated with this sequence of seminorms is the topology defined by the distance

$$
(x, y) \mapsto d(x, y):=\sum_{n=1}^{+\infty} \frac{\inf \left(1,\|x-y\|_{n}\right)}{2^{n}}
$$

A sequence $\left(x_{m}\right)_{m \geq 1}$ converges to $x \in V$ if and only if $\lim _{m \rightarrow+\infty} d\left(x, x_{m}\right)=0$, or, equivalently, if and only if $\lim _{n \rightarrow+\infty}\left\|x-x_{m}\right\|_{n}=0$ for every $n \geq 1$. Similarly a sequence $\left(x_{m}\right)_{m \geq 1}$ of elements of $V$ is a Cauchy sequence if and only if it is a Cauchy sequence with respect to the distance $d$, or, equivalently, if it is a Cauchy sequence with respect to the seminorm $\|\cdot\|_{n}$ for every $n \geq 1$. The space $\left(F,\|\cdot\|_{n}\right)_{n \geq 1}$ is called a Fréchet space when every Cauchy sequence of elements of $F$ is convergent.

Now suppose that $\left(V,\left(\|\cdot\|_{n}\right)_{n \geq 1}\right.$ is a Fréchet space. A set $D \subset V$ is said to be bounded if $\sup _{x \in D}\|x\|_{n}<+\infty$ for every $n \geq 1$ (or, equivalently, if and only if for every neighbourhood $U$ of the origin in $V$ there exists $\lambda_{U}>0$ such that $\left.\lambda_{U} D \subset U\right)$. A linear operator $R: V \rightarrow V$ is said to be bounded if $R(D)$ is bounded for every bounded subset $D$ of $V$, and it follows from a standard elementary result that a linear operator $T: V \rightarrow V$ is continuous with respect to the topology of $V$ if and only if $T$ is bounded. 
Denote by $\mathcal{B}(V)$ the algebra of bounded linear operators $R: V \rightarrow V$. A subset $\Delta$ of $\mathcal{B}(V)$ is said to be bounded if $\bigcup_{R \in \Delta} R(D)$ is bounded for every bounded subset $D$ of $V$. A sequence $\left(R_{m}\right)_{m \geq 1}$ of elements of $\mathcal{B}(V)$ is said to be Mackey-convergent to $R \in \mathcal{B}(V)$ if and only if there exists a sequence $\left(\lambda_{m}\right)_{m \geq 1}$ of positive real numbers such that $\lim _{m \rightarrow+\infty} \lambda_{m}=+\infty$ and such that the set $\left\{\lambda_{m}\left(R-R_{m}\right)\right\}_{m \geq 1}$ is bounded. When $\mathcal{X}$ is a Banach space, a subset $\Delta$ of $\mathcal{B}(\mathcal{X})$ is bounded if and only if $\sup _{R \in \Delta}\|R\|<+\infty$, where $\|R\|=\sup _{\|x\| \leq 1}\|R x\|$ is the usual norm on $\mathcal{B}(\mathcal{X})$, and a sequence $\left(R_{m}\right)_{m \geq 1}$ of elements of $\mathcal{B}(\mathcal{X})$ converges to $R \in \mathcal{B}(\mathcal{X})$ in the sense of Mackey if and only if $\lim _{m \rightarrow+\infty}\left\|R-R_{m}\right\|=0$.

Let $\Omega$ be an open subset of $\mathbb{C}$, and let $V$ be a Fréchet space. A map $\phi: \Omega \rightarrow V$ is said to be analytic if for every $a \in \Omega$ there exists $r>0$ and a sequence $\left(\alpha_{n}\right)_{n \geq 0}$ of elements of $V$ such that $\phi(z)=\sum_{n=0}^{+\infty}(z-a)^{n} \alpha_{n}$ for $z \in D(a, r):=\{z \in \mathbb{C}:|z-a|<r\}$, and a map $\psi: \Omega \rightarrow \mathcal{B}(V)$ is said to be analytic if for every $a \in \Omega$ there exists $\rho>0$ and a sequence $\left(R_{n}\right)_{n \geq 0}$ of elements of $\mathcal{B}(V)$ such that $\psi(z)=\sum_{n=0}^{+\infty}(z-a)^{n} R_{n}$ for $z \in D(a, \rho)$, the series being convergent in the sense of Mackey. A routine verification shows that if $\psi: \Omega \rightarrow \mathcal{B}(V)$ is analytic, then the map $z \rightarrow \psi(z) x$ is analytic for every $x \in V$.

Now let $V_{1}$ and $V_{2}$ be two Fréchet spaces, and let $W$ be a linear subspace of $\mathcal{B}\left(V_{1}\right)$. A linear map $\theta: W \rightarrow \mathcal{B}\left(V_{2}\right)$ is said to be bounded if $\theta(\Delta)$ is a bounded subset of $\mathcal{B}\left(V_{2}\right)$ for every subset $\Delta$ of $W$ which is bounded in $\mathcal{B}\left(V_{1}\right)$. Notice that if $\psi: \Omega \rightarrow W$ is analytic, and if a linear map $\theta: W \rightarrow \mathcal{B}\left(V_{2}\right)$ is bounded, then $\theta \circ \psi: \Omega \rightarrow \mathcal{B}\left(V_{2}\right)$ is analytic.

2. Normalization of analytic semigroups, and quasimultipliers. If $\mathcal{A}$ is a commutative Banach algebra, let $\mathcal{A}^{\perp}=\{x \in \mathcal{A}: x y=0 \quad \forall y \in \mathcal{A}\}$. We begin with an easy observation. We will say that a semigroup $(T(t))_{t>0}$ in a Banach algebra is normalized if $\mathcal{A}_{T}^{\perp}=\{0\}$, where $\mathcal{A}_{T}$ denotes the closed subalgebra generated by the semigroup. The following proposition allows us to "normalize" semigroups.

Proposition 2.1. Let $(T(t))_{t>0}$ be a semigroup in a Banach algebra, and let $\mathcal{A}_{T}$ be the closed algebra generated by the semigroup. Then $\left[\mathcal{A}_{T} / \mathcal{A}_{T}^{\perp}\right]^{\perp}=\{0\}$, and $\mathcal{A}_{T} / \mathcal{A}_{T}^{\perp}$ is not unital if $\mathcal{A}_{T}$ is not unital.

Proof. Let $\pi: \mathcal{A}_{T} \rightarrow \mathcal{A}_{T} / \mathcal{A}_{T}^{\perp}$ be the canonical surjection, let $u \in\left[\mathcal{A}_{T} / \mathcal{A}_{T}^{\perp}\right]^{\perp}$ and let $x \in \mathcal{A}_{T}$ such that $\pi(x)=u$. Let $t>0$. We have $\pi(T(t / 2)) u=0$, so $T(t / 2) x \in \mathcal{A}_{T}^{\perp}$ and $T(t) x=0$. Since $\mathcal{A}_{T}$ is the closed algebra generated by the semigroup $(T(t))_{t>0}$ we have $x \in \mathcal{A}_{T}^{\perp}$, and $u=\pi(x)=0$. Now assume that $\mathcal{A}_{T} / \mathcal{A}_{T}^{\perp}$ is unital, denote by $P$ its unit element, and let $J \in \pi^{-1}(P)$. We have $x-J x \in \mathcal{A}_{T}^{\perp}$ for every $x \in \mathcal{A}_{T}$. In particular $T(t)-T(t) J=T(t / 2)(T(t / 2)-T(t / 2) J)=0$ for every $t>0$, and so $\mathcal{A}_{T}$ admits $J$ as its unit element (which implies that $\mathcal{A}_{T}^{\perp}=0$ ). So the quotient algebra $\mathcal{A}_{T} / \mathcal{A}_{T}^{\perp}$ is not unital if $\mathcal{A}_{T}$ is not unital.

We will say that a semigroup $(T(t))_{t>0}$ of bounded operators in a Banach algebra is norm continuous if $\lim _{h \rightarrow 0}\|T(t+h)-T(t)\|=0$ for every $t>0$. If the closed subalgebra $\mathcal{A}_{T}$ generated by such a semigroup has a unit element $I$, then the ideal $\bigcup_{t>0} T(t) \mathcal{A}_{T}$, which is dense in $\mathcal{A}_{T}$, must equal $\mathcal{A}_{T}$ and $\lim _{t \rightarrow 0^{+}}\|I-T(t)\|=0$. Hence there exists $u \in$ $\mathcal{A}_{T}$ such that $T(t)=e^{t u}$. In other terms the generator of a norm-continuous semigroup $(T(t))_{t>0}$ is bounded if the algebra $\mathcal{A}_{T}$ is unital. 
Recall that if $(T(t))_{t>0}$ is a strongly continuous semigroup of bounded operators on a Banach space $\mathcal{X}$, the infinitesimal generator $\Delta: \mathcal{D}_{\Delta} \rightarrow \mathcal{X}$ of the semigroup is the linear operator defined for $u \in \mathcal{D}_{\Delta}$ by the formula

$$
\Delta(u)=\lim _{t \rightarrow 0^{+}} \frac{T(t) u-u}{t},
$$

where we denote by $\mathcal{D}_{\Delta}$ the set of all $x \in \mathcal{X}$ for which the quotient $\frac{T(t) x-x}{t}$ has a limit as $t \rightarrow 0^{+}$. If the map $t \rightarrow T(t)$ is differentiable with respect to the norm of $\mathcal{B}(\mathcal{X})$, then $T(t) \mathcal{X} \subset \mathcal{D}_{\Delta}$ and we have, for every $t>0$,

$$
T^{\prime}(t)=\Delta T(t) \in \mathcal{B}(\mathcal{X}) .
$$

Recall also a standard procedure due to Feller [10]. Given a strongly continuous semigroup $(T(t))_{t>0}$ of bounded operators on a Banach space $\mathcal{X}$ and an $\epsilon>0$ there is a Banach space $\mathcal{Y}$ and a bounded map $\theta: \mathcal{R} \rightarrow \mathcal{B}(\mathcal{Y})$, where

$$
\mathcal{R}:=\{S \in \mathcal{B}(\mathcal{X}): S T(t)=T(t) S \quad \forall t>0\},
$$

which possesses the following properties:

1. $\|\theta(S)\| \leq\|S\| \forall S \in \mathcal{R}$,

2. $\|\theta(T(t))\| \leq e^{(\rho(T(1))+\epsilon) t} \forall t>0$,

3. the generator of $(\theta(T(t)))_{t>0}$ is unbounded if the generator of $(T(t))_{t>0}$ is unbounded.

In this construction $\mathcal{Y}$ is the space of all $y \in \mathcal{X}$ for which $T(t) x$ has a limit in $(\mathcal{X},\|\|$. as $t \rightarrow 0+$, which is a Banach space with respect to the norm

$$
y \mapsto\|y\|:=\sup _{t>0}\left\|e^{-\nu t} T(t) y\right\|,
$$

where $\nu=\rho(T(1))+\epsilon$; also $S(\mathcal{Y}) \subset \mathcal{Y}$ for every $S \in \mathcal{R}$, and $\theta$ is the restriction map $S \rightarrow \mathcal{S}_{\mid \mathcal{Y}}$. This idea was used by the second author in [9] to construct, for any non-unital commutative Banach algebra having a norm continuous semigroup $(T(t))_{t>0}$ generating a dense ideal, a norm-decreasing homomorphism with dense range in another commutative Banach algebra for which the multiplier algebra has a very rich set of characters. We now apply these methods to Banach algebras generated by semigroups analytic on the half-line or on open sectors.

Definition 2.2. Let $\mathcal{A}$ be a commutative Banach algebra having dense principal ideals such that $\mathcal{A}^{\perp}=\{0\}$. Set $\Omega(\mathcal{A}):=\left\{x \in \mathcal{A}:[x \mathcal{A}]^{-}=\mathcal{A}\right\}$. A quasimultiplier on $\mathcal{A}$ is a fraction $a / b$, where $a \in \mathcal{A}$ and $b \in \Omega(\mathcal{A})$, and the set of quasimultipliers on $\mathcal{A}$ is denoted by $Q M(\mathcal{A})$. If $u=a / b$ is a quasimultiplier on $\mathcal{A}$, we define the domain $\mathcal{D}_{u}$ of $u$ by

$$
\mathcal{D}_{u}:=\{x \in \mathcal{A}: u x \in \mathcal{A}\} .
$$

A set $U \subset Q M(\mathcal{A})$ is said to be pseudobounded if there exists $x \in \Omega(\mathcal{A}) \cap\left[\bigcap_{u \in U} \mathcal{D}_{u}\right]$ such that $\sup _{u \in U}\|x u\|<+\infty$.

We identify as usual $a / b$ with $a^{\prime} / b^{\prime}$ when $a b^{\prime}-b a^{\prime}=0$. Since $\Omega(\mathcal{A})$ is stable under multiplication, $Q M(\mathcal{A})$ is an algebra with respect to the usual operations on fractions, and a finite union of pseudobounded sets is pseudobounded. Also the map $(\lambda, x) \mapsto \lambda x$ is a bounded map from $\mathbb{C} \times Q M(\mathcal{A})$ into $Q M(\mathcal{A})$, and the maps $(x, y) \mapsto x+y$ and 
$(x, y) \mapsto x y$ are bounded maps from $Q M(\mathcal{A}) \times Q M(\mathcal{A})$ into $Q M(\mathcal{A})$, which means that $Q M(\mathcal{A})$ is a "bornological algebra". We denote by $I$ the unit element of $Q M(\mathcal{A})$. We now introduce an important subalgebra of $Q M(\mathcal{A})$.

Definition 2.3. Let $\mathcal{A}$ be a commutative Banach algebra having dense principal ideals such that $\mathcal{A}^{\perp}=\{0\}$. A quasimultiplier $r$ on $\mathcal{A}$ is said to be regular if there exists $\lambda>0$ such that the set $\left\{\lambda^{n} r^{n}\right\}_{n \geq 1}$ is pseudobounded in $Q M(\mathcal{A})$, and the set of regular quasimultipliers on $\mathcal{A}$ is denoted by $Q M_{r}(\mathcal{A})$. A set $V \subset Q M_{r}(\mathcal{A})$ is said to be multiplicatively pseudobounded (or m-pseudobounded) if there exists a pseudobounded set $U$ in $\mathcal{A}$ which is stable under products and $\epsilon>0$ such that $\epsilon V \subset U$.

Routine verifications show that $Q M_{r}(\mathcal{A})$ is indeed an algebra, that the map $(\lambda, x) \mapsto$ $\lambda x$ is a bounded map from $\mathbb{C} \times Q M_{r}(\mathcal{A})$ into $Q M_{r}(\mathcal{A})$, and that the maps $(x, y) \mapsto x+y$ and $(x, y) \mapsto x y$ are bounded maps from $Q M_{r}(\mathcal{A}) \times Q M_{r}(\mathcal{A})$ into $Q M_{r}(\mathcal{A})$, which means that $Q M_{r}(\mathcal{A})$ is also a "bornological algebra". Now set

$$
M(\mathcal{A}):=\{R \in \mathcal{B}(\mathcal{A}): \operatorname{Rax}=a R x \forall a, x \in \mathcal{A}\},
$$

and fix $u \in \Omega(\mathcal{A})$. The map $\theta: R \rightarrow R u / u$ is clearly an injective bounded homomorphism from $M(\mathcal{A})$ into $Q M_{r}(\mathcal{A})$. In the sequel we will identify $M(\mathcal{A})$ with $\theta(M(\mathcal{A}))$, and we set $\|R\|_{M(\mathcal{A})}=\sup _{a \in \mathcal{A} \backslash\{0\}} \frac{\|R a\|}{\|a\|}$. Part of the following result, based on Feller's method, was proved in [9, p. 119].

Lemma 2.4. Let $\mathcal{A}$ be a commutative Banach algebra having dense principal ideals such that $\mathcal{A}^{\perp}=\{0\}$, and let $V$ be a pseudobounded set in $Q M(\mathcal{A})$. Set

$$
J=\left\{x \in \mathcal{A} \mid x V \subset \mathcal{A} \text {, and }\|x\|_{J}:=\sup _{v \in V \cup\{I\}}\|x v\|<+\infty\right\} .
$$

Then $J$ is a dense ideal of $\mathcal{A}$ which is also a Banach $\mathcal{A}$-module with respect to the norm $\|.\|_{J}$, and $\Omega(\mathcal{A}) \cap J \neq \emptyset$. Moreover, ux $\in J$ for every $u \in M(\mathcal{A})$, and we have

1. $\|x\|_{J} \geq\|x\| \quad \forall x \in \mathcal{A}$.

2. $\|a x\|_{J} \leq\|a\|\|x\|_{J} \quad \forall a \in \mathcal{A}, \forall x \in J$.

3. $\|u x\|_{J} \leq\|u\|_{M(\mathcal{A})}\|x\|_{J} \quad \forall u \in M(\mathcal{A}), \forall x \in J$.

Also, if we denote by $J_{0}$ the closure of $\operatorname{span}\{a x\}_{a \in \mathcal{A}, x \in J}$ in $\left(J,\|\cdot\|_{J}\right)$, then $J_{0}$ is dense in $\mathcal{A}, \Omega(\mathcal{A}) \cap J \neq \emptyset, x^{2} \in J_{0}$, and $x^{2} J_{0}$ is dense in $\left(J_{0},\|\cdot\|_{J}\right)$ for every $x \in \Omega(\mathcal{A}) \cap J$.

Further, if $V$ is stable under products, then $v J \subset J, v J_{0} \subset J_{0}$ for $v \in V$, and we have

$$
\|v x\|_{J} \leq\|x\|_{J} \quad \forall v \in V, \forall x \in J .
$$

Proof. This lemma is proved in [9] for pseudobounded sets stable under products, but we give a proof for the sake of completeness. It is clear that $J$ is an ideal of $\mathcal{A}$, and that 1 . and 2. hold. Now let $u \in M(\mathcal{A})$, let $x \in J$, and let $v \in V \cup\{I\}$. We have $(u x) v=u(x v) \in \mathcal{A}$, and $\|(u x) v\|=\|u(x v)\| \leq\|u\|_{M(\mathcal{A})}\|x v\|$, which shows that $u x \in J$ and $\|u x\|_{J} \leq\|u\|\|x\|_{J}$.

Let $\left(x_{n}\right)_{n \geq 0}$ be a Cauchy sequence in $\left(J,\|\cdot\|_{J}\right)$. Then $\left(x_{n}\right)_{n \geq 0}$ is a Cauchy sequence in $\mathcal{A}$, and so there exists $x \in \mathcal{A}$ such that $\lim _{n \rightarrow+\infty}\left\|x-x_{n}\right\|=0$. Let $\epsilon>0$, and let $N \geq 1$ such that $\left\|x_{p}-x_{q}\right\|_{J}<\frac{\epsilon}{2}$ for $p \geq N, q \geq N$. Fix $p \geq N$, and let $v \in V \cup\{1\}$. We have $\left\|\left(x-x_{p}\right) v\right\|=\lim _{q \rightarrow+\infty}\left\|\left(x_{p}-x_{q}\right) v\right\| \leq \lim \sup _{q \rightarrow+\infty}\left\|x_{p}-x_{q}\right\|_{J} \leq \frac{\epsilon}{2}<\epsilon$, and so $\lim _{p \rightarrow \infty}\left\|x-x_{p}\right\|_{J}=0$, so that $\left(J,\|\cdot\|_{J}\right)$ is a Banach space. 
It follows from the definition of pseudobounded sets that $\Omega(\mathcal{A}) \cap J \neq \emptyset$. Let $x \in$ $\Omega(\mathcal{A}) \cap J$. Then $x^{2} \in J_{0} \cap \Omega(\mathcal{A})$, so that $J_{0}$ is dense in $\mathcal{A}$. Now let $a \in \mathcal{A}$, and let $y \in J$. There exists a sequence $\left(u_{n}\right)_{n \geq 1}$ of elements of $\mathcal{A}$ such that $\lim _{n \rightarrow+\infty}\left\|a-x^{2} u_{n}\right\|=0$. Since $\left\|a y-x^{2} u_{n} y\right\|_{J} \leq\left\|a-x^{2} u_{n}\right\|\|y\|_{J}$, we have $\lim _{n \rightarrow+\infty}\left\|a y-x^{2} u_{n} y\right\|_{J}=0$, which shows that $x^{2} J_{0}$ is dense in $\left(J_{0},\|\cdot\|_{J}\right)$.

Now assume that $V$ is stable under products, and let $v \in V$. If $x \in J$, then $v x V=$ $x v V \subset x V \subset \mathcal{A}$, so that $v x \in J$, and we have

$$
\|v x\|_{J}=\sup _{w \in V}\|v x w\|=\sup _{w \in V}\|x v w\| \leq \sup _{w \in V}\|x w\|=\|x\|_{J} .
$$

If $a \in \mathcal{A}, y \in J, v \in V$, then $v a y=a v y \in J_{0}$. Since $\operatorname{span}\{a y\}_{a \in \mathcal{A}, y \in V}$ is dense in $\left(J_{0},\|\cdot\|_{J}\right)$, we have $v J_{0} \subset J_{0}$ for every $v \in V$.

Notice that the ideal $J$ above is also a Banach algebra with respect to the norm $\|\cdot\|_{J}$. An easy example given in [9, p. 137] shows that we may have $\Omega(J)=\emptyset$ if an ideal $J$ of a commutative Banach algebra $\mathcal{A}$ is complete with respect to a norm $\|\cdot\|_{J}$ satisfying 1 . and 2. even if $J \cap \Omega(\mathcal{A}) \neq \emptyset$, so that $J_{0}$ may be strictly contained in $J$.

The algebra $Q M_{r}(\mathcal{A})$ is a "pseudo-Banach algebra" in the sense of Allan, Dales and McClure [1], which means that it is the inductive limit of a family of Banach algebras. To see this denote by $\mathcal{V}$ the set of all pseudobounded subsets of $Q M(\mathcal{A})$ which are stable under products and contain the unit element $I$ of $Q M(\mathcal{A})$. For $V_{1}, \ldots, V_{k}$ in $\mathcal{V}$ denote by $V_{1} \ldots V_{k}$ the set of all products of the form $x=x_{1} x_{2} \ldots x_{k}$, where $x_{j} \in V_{J}$ for $1 \leq j \leq k$. Then $V_{1} \ldots V_{k}$ is a pseudobounded set containing $\bigcup_{1 \leq j \leq k} V_{j}$ which is stable under products, and we see that the family $\mathcal{V}$ forms an inductive set with respect to inclusion. Denote by $J_{0}(V)$ the ideal $J_{0}$ of $\mathcal{A}$ associated with $V \in \mathcal{V}$ as in Lemma 2.4 and set $\|u\|_{\mathrm{op}, V}=\sup _{x \in J_{0}(V) \backslash\{0\}} \frac{\|u x\|_{J}}{\|x\|_{J}}$ for $u \in M\left(J_{0}\right)$, where the multiplier algebra $M\left(J_{0}\right)$ is identified with the set $\left\{u \in Q M_{r}(\mathcal{A}): u J_{0} \subset J_{0}\right\}$. We obtain the following description of $Q M_{r}(\mathcal{A})$ as a pseudo-Banach algebra (which seems somewhat more natural than the description proposed in [9]).

Proposition 2.5. Let $\mathcal{A}$ be a commutative Banach algebra having dense principal ideals such that $\mathcal{A}^{\perp}=\{0\}$. Then we have, with respect to inclusion

$$
Q M_{r}(\mathcal{A})=\lim _{\overrightarrow{\mathcal{V}}} M\left(J_{0}(V)\right)
$$

More precisely, $Q M_{r}(\mathcal{A})=\bigcup_{V \in \mathcal{V}} M\left(J_{0}(V)\right)$, and a subset $U$ of $Q M_{r}(\mathcal{A})$ is m-pseudobounded if and only if there exists $V$ in $\mathcal{V}$ such that $U \subset M\left(J_{0}(V)\right)$ and $\sup _{u \in U}\|u\|_{\mathrm{op}, V}$ $<+\infty$.

In fact the algebras $M\left(J_{0}(V)\right)$ can also be identified with the multiplier algebras $M\left(\mathcal{A}_{V}\right)$, where we denote by $\mathcal{A}_{V}$ the closure of $J_{0}$ in $M\left(J_{0}\right)$ with respect to the norm $\|.\|_{\text {op }, J_{0}}$. The algebra $\bigcup_{V \in \mathcal{V}} \mathcal{A}_{V}$ is the Mackey closure of $\mathcal{A}$ in $Q M_{r}(\mathcal{A})$, i.e., the set of all $b \in Q M_{r}(\mathcal{A})$ for which there exists a sequence $\left(a_{n}\right)_{n \geq 1}$ of elements of $\mathcal{A}$ and a sequence $\lambda_{n}$ of positive real numbers, with $\lim _{n \rightarrow+\infty} \lambda_{n}=+\infty$, such that the sequence $\left(\lambda_{n}\left(a-a_{n}\right)\right)_{n \geq 1}$ is $m$-pseudobounded. The character space $\widehat{Q M_{r}(\mathcal{A})}$ of $Q M_{r}(\mathcal{A})$, which 
is compact, satisfies

$$
\left.\widehat{Q M_{r}(\mathcal{A})}=\underset{\overleftarrow{\mathcal{V}}}{\lim }\left(\widehat{M\left(J_{0}(V)\right.}\right), \phi_{V, W}\right)
$$

where $\phi_{V, W}(\chi)=\phi_{\mid M\left(J_{0}(V)\right)}$ for $V, W \in \mathcal{V}, V \subset W, \phi \in M \widehat{\left(J_{0}(W)\right)}$.

One of the main results of [9] is that $\widehat{Q M_{r}(\mathcal{A})}$ is very rich if $\mathcal{A}$ contains a normcontinuous semigroup $(T(t))_{t>0}$ such that $\bigcup_{t>0} T(t) \mathcal{A}$ is dense in $\mathcal{A}$ : in this situation there exists a surjective continuous mapping

$$
\psi: \widehat{H^{\infty}(\mathbb{D})} \rightarrow \widehat{Q M_{r}(\mathcal{A})},
$$

where $H^{\infty}(\mathbb{D})$ is the algebra of bounded holomorphic functions on the open unit disc $\mathbb{D}$.

We conclude this section with an elementary but useful observation, to be used later on, which is not contained in 9 .

Proposition 2.6. Let $\mathcal{A}$ be a commutative Banach algebra such that $\Omega(\mathcal{A}) \neq \emptyset$ and such that $\mathcal{A}^{\perp}=\{0\}$, and let $\left(e_{p}\right)_{p \geq 1}$ be a sequence of elements of $\mathcal{A}$ with $\lim _{p \rightarrow+\infty}\left\|x-x e_{p}\right\|=0$ for some $x \in \Omega(\mathcal{A})$. Set

$$
H=\left\{u \in Q M(\mathcal{A}): u e_{p} \in \mathcal{A} \forall p \geq 1, \text { and }\|u\|_{H}:=\sup _{p \geq 1}\left\|u e_{p}\right\|<+\infty\right\},
$$

and denote by $H_{0}$ the closure of $\operatorname{span}\{a u\}_{a \in \mathcal{A}, u \in H}$ in $\left(H,\|\cdot\|_{H}\right)$.

Then $H \subset M(\mathcal{A})$, and $H_{0} \subset \mathcal{A}$. Also $\lim _{p \rightarrow+\infty}\left\|u e_{p} a-u a\right\|=0$ for every $a \in \mathcal{A}$ if $u \in H$, and $\lim _{p \rightarrow+\infty}\left\|u e_{p}-u\right\|=0$ if $u \in H_{0}$.

Proof. Let $u \in H$, and let $a \in \mathcal{A}$ and $b \in \Omega(\mathcal{A})$ such that $u=a / b$. The sequence $\left(u e_{p}\right)_{p \geq 1}$ is bounded, and

$$
\lim _{p \rightarrow+\infty}\left\|u e_{p} x b y-a x y\right\|=\lim _{p \rightarrow+\infty}\left\|a y\left(x e_{p}-x\right)\right\|=0
$$

for every $y \in \mathcal{A}$. Since the sequence $\left(u e_{p}\right)_{p \geq 1}$ is bounded, and since $x b \mathcal{A}$ is dense in $\mathcal{A}$, this shows that the sequence $\left(u e_{p} v\right)_{p \geq 1}$ has a limit $\phi(v)$ in $\mathcal{A}$ for every $v \in \mathcal{A}$, and $\phi(y v)=y \phi(v)$ for every $y \in \mathcal{A}$. We have $b x \phi(v)=\phi(x b v)=a x v$, so that $b \phi(v)=a v$. Hence $u v=\phi(v) \in \mathcal{A}$ for every $v \in \mathcal{A}$, and $H \subset M(\mathcal{A})$.

If $a \in \mathcal{A}, y \in H$, then $\lim _{p \rightarrow+\infty}\left\|a y e_{p}-a y\right\|=0$. Hence $\lim _{p \rightarrow+\infty}\left\|v e_{p}-v\right\|=0$ for every $v \in \operatorname{span}\{a y\}_{a \in \mathcal{A}, y \in H}$.

Now let $u$ be an element of the closure of $\operatorname{span}\{a y\}_{a \in \mathcal{A}, y \in H}$ in $\left(H,\|\cdot\|_{H}\right)$. We have, for $v \in \operatorname{span}\{a y\}_{a \in \mathcal{A}, y \in H}$ in $\left(H,\|\cdot\|_{H}\right)$,

$$
\left\|u e_{p}-u e_{q}\right\| \leq 2\|u-v\|_{H}+\left\|v e_{p}-v e_{q}\right\| .
$$

It follows from this inequality that the sequence $\left(u e_{p}\right)_{p \geq 1}$ is a Cauchy sequence in $\mathcal{A}$. Since $\lim _{p \rightarrow+\infty}\left\|a u e_{p}-a u\right\|=0$ for every $a \in \mathcal{A}$, we see that in fact $\lim _{p \rightarrow+\infty}\left\|u e_{p}-u\right\|=0$, and so $u \in \mathcal{A}$.

3. Boundary values of sectorial semigroups. We now consider a normalized analytic semigroup $(T(t))_{t>0}$ in a Banach algebra $\mathcal{A}$, and we assume that the algebra generated by the semigroup is dense in $\mathcal{A}$ and that the generator $\Delta$ of the semigroup is unbounded, which is equivalent to the fact that $\mathcal{A}$ is not unital. It follows from the analyticity of the semigroup that $T(t) \in \Omega(\mathcal{A})$ for $t>0$, and so $\Delta=T^{\prime}(t) / T(t) \in Q M(\mathcal{A})$. 
The formula

$$
T(0)=I, \quad \text { and } \quad T(-t)=1 / T(t) \text { for } t>0
$$

defines an extension of the semigroup to a group $T(t)_{t \in \mathbb{R}}$ of quasimultipliers. Set $L=$ $\bigcap_{t>0} T(t) \mathcal{A}$, and set $\|u\|_{n}=\max _{0 \leq m \leq n}\|u / T(n)\|$ for $u \in L, n \geq 0$. Then $\left(L,\|\cdot\|_{n}\right)_{n \geq 0}$ is a Fréchet space and an ideal of $\mathcal{A}$, and we have, for $a \in \mathcal{A}, u \in L, n \geq 0$,

$$
\|a u\|_{n} \leq\|a\|\|u\|_{n} .
$$

A routine verification shows that $(T(t))_{t \in \mathbb{R}}$ is a group of bounded operators on this Fréchet space. The interest of this observation is limited, since the quasimultipliers $T(-t)$ cannot be regular for $t>0$, for otherwise there would be a Banach algebra $\mathcal{B} \subset Q M(\mathcal{A})$ similar to $\mathcal{A}$ in the sense of [9] in which the generator of the semigroup would be bounded, which would force $\mathcal{B}$, and hence $\mathcal{A}$, to be unital. A similar argument shows that the quasimultiplier $\Delta$ is not regular, and so the map $t \mapsto T(t)$, mapping $\mathbb{R} \rightarrow \mathcal{B}(L)$, is not analytic at $t$ for any $t \leq 0$.

REMARK 3.1. $\Omega\left(\mathcal{A}_{T}\right) \cap \bigcap_{t>0} T(t) \mathcal{A}_{T}$, is dense in $\Omega(\mathcal{A})$. In particular there exists $x \in$ $\Omega\left(\mathcal{A}_{T}\right)$ such that the map $z \rightarrow T(z) x$ admits an analytic extension to $\mathbb{C}$.

Proof. We know that $\Omega(\mathcal{A})$ is a dense $G_{\delta}$-set, i.e., a countable intersection of dense open subsets of $\mathcal{A}_{T}$. So the relative topology on $\Omega\left(\mathcal{A}_{T}\right)$ can be defined by a distance $d$ on $\Omega\left(\mathcal{A}_{T}\right)$ with respect to which $\Omega\left(\mathcal{A}_{T}\right)$ is a complete metric space. Since $T(t) \in \Omega\left(\mathcal{A}_{T}\right)$, for every $t \in \mathcal{S}_{\alpha}$, we see that $T(1) \Omega$ is dense in $\Omega\left(\mathcal{A}_{T}\right)$, and it follows from the Mittag-Leffler theorem on inverse limits [8] that $\bigcap_{t>0} T(t) \mathcal{A}_{T}=\bigcap_{n>1} T(1)^{n} \Omega\left(\mathcal{A}_{T}\right)$ is dense in $\Omega\left(\mathcal{A}_{T}\right)$.

Let $x \in \Omega(\mathcal{A}) \cap \bigcap_{t>0} T(t) \mathcal{A}_{T}$, and set $F(z)=T(z) x$ for $z \in \mathbb{C}$. Let $a \in \mathbb{C}$. Since there exists $m \geq 1$ such that $a+m \in \mathcal{S}_{\alpha}$, we have $F^{(n)}(a)=\Delta^{n} T(a) x$ for $n \geq 0$, and

$$
\lim _{n \rightarrow+\infty}\left\|T(z) x-\sum_{p=0}^{n} \frac{(z-a)^{p}}{p !} T(a) \Delta^{p}(x)\right\|=0
$$

for every $z \in \mathbb{C}$. In other words the map $z \rightarrow T(z)$ is an analytic map in the sense of Mackey from $\mathbb{C}$ into $Q M\left(\mathcal{A}_{T}\right)$ with respect to the family of pseudobounded sets.

We shall see later that $\limsup _{n \rightarrow \infty}\|T(-n \delta) x\|^{\frac{1}{n}}=+\infty$ for every $n \geq 0$ and every $x \in \Omega(A) \cap \bigcap_{t>0} T(t) \mathcal{A}_{T}$, and that $\lim _{\sup _{n \rightarrow+\infty}}\left\|\Delta^{n} x\right\|^{\frac{1}{n}}=+\infty$ for every $x \in \operatorname{Dom}\left(\Delta^{n}\right) \cap$ $\Omega\left(\mathcal{A}_{T}\right)$. Notice that this is not true if we consider $\mathcal{A}_{T} \backslash\{0\}$ instead of $\Omega\left(\mathcal{A}_{T}\right)$ : set $T(t)(u)$ $=u^{t}$ for $u \in[0,1], t \in \mathcal{S}_{\frac{\pi}{2}}$. Then $(T(t))_{t \in \mathcal{S}_{\frac{\pi}{2}}}$ is an analytic semigroup in $\mathcal{C}_{0}([0,1]):=$ $\{f \in \mathcal{C}([0,1]): f(0)=0\}$.

Now fix $\delta \in[0,1)$, and set $I_{\delta}:=\left\{f \in \mathcal{C}_{0}([0,1]): f(u)=0 \forall u \in[0, \delta]\right\}$. Denote by $\Delta$ the infinitesimal generator of the semigroup, so that $\Delta(f)(u)=\log (u) f(u)$ for $f \in \mathcal{C}_{0}([0,1])$. We have, for every $\delta>0$ and every $f \in I_{\delta}$,

$$
\limsup _{n \rightarrow \infty}\|T(-n \delta) x\|^{\frac{1}{n}}<+\infty \quad \text { and } \quad \limsup _{n \rightarrow+\infty}\left\|\Delta^{n} x\right\|^{\frac{1}{n}}<+\infty,
$$

which just means that the generator of the restriction of the semigroup to $I_{\delta}$ is bounded.

We consider now a semigroup $(T(t))_{t \in S_{\alpha}}$ of bounded operators on a Banach space $\mathcal{X}$, which is analytic on the open sector. We will denote by $\mathcal{A}_{T}$ the closed subalgebra 
generated by the semigroup. We will assume that $\bigcup_{t>0} T(t) \mathcal{X}$ is dense in $\mathcal{X}$ (if this is not the case replace $\mathcal{X}$ by $\mathcal{Y}=\left[\bigcup_{t>0} T(t) \mathcal{X}\right]^{-}$).

In this context we may mention the Kato-Neuberger theorem [13] (see also [4]), to the effect that if for a semigroup $(T(t))_{t>0}$ in a unital algebra with unit $I$ there are $\epsilon, \delta>0$ such that $\|T(t)-I\| \leq 2-\epsilon$ for $0<t<\delta$, then the semigroup is analytic in some sector.

For $a \in \mathbb{C}$ and $r>0$ we set

$$
D(a, r)=\{z \in \mathbb{C}:|z-a|<r\} \quad \text { and } \quad D^{+}(a, r)=\{z \in D(a, r): \operatorname{Re} z>\operatorname{Re} a\} .
$$

REMARK 3.2. Analytic semigroups bounded near the origin can be extended to the closed sector. Assume that there exists $r>0$ such that

$$
\sup _{t \in D(0, r) \cap S_{\alpha}}\|T(t)\|<+\infty .
$$

Then $\lim _{\substack{t \rightarrow S_{\alpha} \\ t \in S_{\alpha}}} T(t) x$ exists for every $x \in \mathcal{X}$ and every $w \in \partial S_{\alpha}$. Moreover if we set

$$
T(w)=\lim _{\substack{t \rightarrow w \\ t \in S_{\alpha}}} T(t) x,
$$

then $(T(t))_{t \in \overline{S_{\alpha}}}$ is a semigroup of bounded operators which is continuous with respect to the strong operator topology. For we have $\lim _{\substack{t \rightarrow w \\ t \in S_{\alpha}}} T(t) T\left(t_{0}\right) x=T\left(t_{0}\right) x$ for every $t_{0}>0$ and every $x \in \mathcal{X}$. Now the result follows immediately from the fact that $\bigcup_{t>0} T(t) \mathcal{X}$ is dense in $\mathcal{X}$, given that $\sup _{z \in D(0, r) \cap S_{\alpha}}\|T(t)\|<+\infty$.

Now consider a normalized semigroup $(T(t))_{t \in \mathcal{S}_{\alpha}}$ in a Banach algebra $\mathcal{A}$, with unbounded generator, and assume that the algebra generated by the semigroup is dense in $\mathcal{A}$, so that $\mathcal{A}$ is not unital. It follows again from the analyticity of the semigroup that $T(t) \mathcal{A}$ is dense in $\mathcal{A}$ for every $t \in \mathcal{S}_{\alpha}$. For every $z \in \mathbb{C}$ there exists a positive integer $p$ such that $p+z \in \mathcal{S}_{\alpha}$, and so $T(z)$ can be defined as a quasimultiplier on $\mathcal{A}$ by the formula

$$
T(z)=\frac{T(p+z)}{T(p)} .
$$

Then $(T(z))_{z \in \mathbb{C}}$ is a group of elements of $Q M(\mathcal{A})$, and $T(z)$ is a bounded operator on the Fréchet space $L=\bigcap_{t>0} T(t) \mathcal{A}$ for $z \in \mathbb{C}$. Again, the interest of this formal extension seems rather limited. We propose first another construction of a larger Fréchet space $K$ containing $\bigcap_{t>0} T(t) \mathcal{A}$ for which the semigroup has "boundary values" on $\partial \mathcal{S}_{\alpha}$ with respect to the strong operator topology on $K$, but for which $T(t)$ is not invertible in $\mathcal{B}(K)$ for $t \in \mathcal{S}_{\alpha}$.

For $\alpha \in\left(0, \frac{\pi}{2}\right]$ and $n \geq 1$, let $\mathcal{S}_{\alpha, n}=\left\{z \in \mathcal{S}_{\alpha}:|z| \leq n\right\}$.

Proposition 3.3. Let $(T(t))_{t \in \mathcal{S}_{\alpha}}$ be a normalized analytic semigroup in a Banach algebra, so that the closed subalgebra $\mathcal{A}:=\mathcal{A}_{T}$ generated by the semigroup satisfies $\mathcal{A}^{\perp}=\{0\}$. For $n \geq 1$, set

$$
J_{n}:=\left\{x \in \mathcal{A}: \sup _{t \in \mathcal{S}_{\alpha, n}}\|T(t) x\|<+\infty\right\},
$$

set $\|x\|_{n}=\max \left(\|x\|, \sup _{t \in \mathcal{S}_{\alpha, n}}\|T(t) x\|\right)$ for $x \in J_{n}$, and denote by $J_{0, n}$ the closure of $T(1) J_{n}$ in $\left(J_{n},\|\cdot\|_{n}\right)$.

(i) The ideal $J_{0}:=\bigcap_{n \geq 1} J_{0, n}$, which contains $\bigcup_{t \in \mathcal{S}_{\alpha}} T(t) \mathcal{A}$, is a Fréchet space with respect to the family $\left(\|\cdot\|_{n}\right)_{n \geq 1}, T(t) J_{0}$ is dense in $\left(J_{0},\left(\|\cdot\|_{n}\right)_{n \geq 1}\right)$ for every $t \in \mathcal{S}_{\alpha}$, 
$u x \in J_{0}$ for every $x \in J_{0}$ and every $u \in M(\mathcal{A})$, and if we set $\tilde{u}(x)=u x$ for $x \in J_{0}$, $u \in M(\mathcal{A})$, then the map $u \mapsto \tilde{u}$ is a bounded linear map from $M(\mathcal{A})$ into $\mathcal{B}\left(J_{0}\right)$.

(ii) We have $T(\zeta) J_{0} \subset J_{0}$ for every $\zeta \in \partial \mathcal{S}_{\alpha}$ and if we set $\tilde{T}(t)(x)=T(t) x$ for $x \in J_{0}$, $t \in \overline{\mathcal{S}}_{\alpha}$, then $(\tilde{T}(t))_{t \in \overline{\mathcal{S}}_{\alpha}}$ is a strongly continuous semigroup of bounded operators on $J_{0}$ (and $(\tilde{T}(t))_{t \in S_{\alpha}}$ is an analytic semigroup of bounded operators on $J_{0}$ ).

Proof. Since $T(1) J_{n}$ is dense in $\operatorname{span}\{a y\}_{a \in \mathcal{A}, y \in J_{n}}$ with respect to the norm $\|\cdot\|_{n}$, the ideals $J_{n}$ and $J_{0, n}$ and the norm $\|\cdot\|_{n}$ are the ideals and the norm associated with the pseudobounded set $\{T(t)\}_{t \in \mathcal{S}_{\alpha, n}}$ in Lemma 2.4. So it follows from Lemma 2.4 that $\left(J_{0, n},\|\cdot\|_{n}\right)$ is an ideal of $\mathcal{A}$ and a Banach $\mathcal{A}$-module for every $n \geq 1$, and so $J_{0}$, which is an ideal of $\mathcal{A}$, is a Fréchet space with respect to the family $\left(\|\cdot\|_{n}\right)_{n \geq 1}$.

Let $x \in J_{0} \cap \Omega(\mathcal{A})$. It follows from Lemma 2.4 that $x^{2} J_{0, n} \subset J_{0, n}$, and that $x^{2} J_{0, n}$ is dense in $\left(J_{0, n},\|\cdot\|_{n}\right)$ for $n \geq 1$. Also $x^{2} \in J_{0}$.

Now let $u \in J_{0}$. There exists for each $n \geq 1$ some $v_{n} \in J_{0, n}$ such that $\left\|u-x^{4} v_{n}\right\|_{n} \leq \frac{1}{n}$. Set $w_{n}=x^{2} v_{n}$. Then $w_{n} \in J_{0}$, and $\lim _{n \rightarrow+\infty}\left\|u-x^{2} w_{n}\right\|_{p}=0$ for every $p \geq 1$, which shows that $x^{2} J_{0}$ is dense in $\left(J_{0},\left(\|\cdot\|_{n}\right)_{n \geq 1}\right)$. In particular $T(t)=T(t / 2)^{2} \in J_{0}$, and $T(t) J_{0}$ is dense in $\left(J_{0},\left(\|\cdot\|_{n}\right)_{n \geq 1}\right)$ for every $t \in \mathcal{S}_{\alpha}$. It follows also from Lemma 2.4 that $u J_{0, n} \subset J_{0, n}$ for every $u \in M(\mathcal{A})$, and that $\|u x\|_{n} \leq\|u\|_{M(\mathcal{A})}\|x\|_{n}$ for every $x \in J_{0, n}$. Hence $u J_{0} \subset J_{0}$ for $u \in M(\mathcal{A})$, and a trivial verification shows that the map $u \mapsto \tilde{u}$ is a bounded map from $M(\mathcal{A})$ into $\mathcal{B}\left(J_{0}\right)$. Since the map $u \mapsto \tilde{u}$ is a bounded map from $M(\mathcal{A})$ into $\mathcal{B}\left(J_{0}\right)$, the semigroup $(T(t))_{t \in \mathcal{S}_{\alpha}}$ is analytic in $\mathcal{B}\left(J_{0}\right)$.

Let $\zeta \in \overline{\mathcal{S}_{\alpha, n}}$, let $n \geq 1$, let $p \geq|\zeta|$ and let $x \in J_{2 n}$. Since $t+\zeta \in \mathcal{S}_{\alpha, n+p}$ for every $t \in \mathcal{S}_{\alpha, n}$, we have $T(t) T(\zeta) x \in \mathcal{A}$ for every $t \in \mathcal{S}_{\alpha, n}$ and

$$
\sup _{t \in \mathcal{S}_{\alpha, n}}\|T(t) T(\zeta) x\| \leq \sup _{s \in \mathcal{S}_{\alpha, 2 n}}\|T(s) x\| \leq\|x\|_{2 n} .
$$

Applying Proposition 2.6 to $T(\zeta) x$ and to the sequence $\left(T\left(\frac{1}{p}\right)\right)_{p \geq 1}$ we see that $T(\zeta) x \in$ $M(\mathcal{A})$ for $\zeta \in \overline{\mathcal{S}_{\alpha, n}}, x \in J_{2 n}$, that $T(\zeta) x \in J_{0, n}$ for $\zeta \in \overline{\mathcal{S}_{\alpha, n}}, x \in J_{0,2 n}$, and that $T(\zeta): J_{0,2 n} \rightarrow J_{0, n}$ is contractive. Hence $T(\zeta)\left(J_{0}\right) \subset J_{0}$ and $\tilde{T}(\zeta) \in \mathcal{B}\left(J_{0}\right)$ for every $\zeta \in \overline{\mathcal{S}_{\alpha}}$.

The map $t \rightarrow T(t) T(1) x$ is clearly a continuous map from $\overline{\mathcal{S}_{\alpha}}$ into $J_{0,2 n}$ for every $x \in J_{2 n}$. Since $T(1) J_{2 n}$ is dense in $\left(J_{0,2 n},\|\cdot\|_{n}\right)$, it follows from (1) that the map $t \rightarrow T(t) x$ is a continuous map from $\overline{\mathcal{S}_{\alpha, n}}$ into $\left(J_{0, n},\|\cdot\|_{n}\right)$ for every $x \in J_{0,2 n}$ and every $n \geq 1$. Hence $(\tilde{T}(t))_{t \in S_{\alpha, n}}$ is strongly continuous on $J_{0}$.

The next proposition links various properties of the semigroup and its boundary values. From now we shall identify $\tilde{T}(t)$ with $T(t)$ for simplicity of notation.

Proposition 3.4. Let $T(t)_{t \in \overline{\mathcal{S}_{\alpha}}}$ be a normalized analytic semigroup. Consider the following properties of the semigroup.

1. There exist $\lambda>0$ and $\delta>0$ such that $\left\{\lambda^{n} T\left(n \delta e^{i \alpha}\right): n \geq 0\right\}$ is bounded in $\mathcal{B}\left(J_{0}\right)$;

2. there exist $\lambda>0$ and $\delta>0$ such that $\left\{\lambda^{n} T\left(n \delta e^{i \alpha}+\eta\right): n \geq 0\right\}$ is bounded in $\mathcal{A}$ for all $\eta \in S_{\alpha}$;

3. there exists $\mu \in \mathbb{R}$ such that for all $\eta \in S_{\alpha}$, one has

$$
\sup _{t>0} e^{\mu t}\left\|T\left(\eta+t e^{i \alpha}\right)\right\|<\infty
$$


4. there exists $\delta>0$ such that $T\left(\delta e^{i \alpha}\right)$ is in $Q M_{r}(\mathcal{A})$;

5. there exists $\mu \in \mathbb{R}$ such that $\left\{e^{\mu t} T\left(t e^{i \alpha}\right): t>0\right\}$ is pseudobounded.

Then $1 . \Rightarrow 2$. $\Leftrightarrow 3$. $\Rightarrow 4$. $\Leftrightarrow 5$.

Proof. 1. $\Rightarrow 2 .:$ this follows immediately from the fact that $T(\eta)=T(\eta / 2)^{2} \in J_{0}$ for every $\eta \in S_{\alpha}$.

2. $\Rightarrow 3$.: given $\eta \in S_{\alpha}$, write $\eta=\eta_{0}+s e^{i \alpha}$, where $\eta_{0}>0$ and $s \in \mathbb{R}$. For $t$ sufficiently large, one can write

$$
\eta+t e^{i \alpha}=\left(\eta_{0} / 2+n \delta e^{i \alpha}\right)+\left(\eta_{0} / 2+(s+t-n \delta) e^{i \alpha}\right),
$$

with $(s+t-n \delta) \in[0, \delta]$. Then

$$
\left\|T\left(\eta+t e^{i \alpha}\right)\right\|_{\mathcal{A}} \leq C_{1} \lambda^{-n} \times C_{2},
$$

for constants $C_{1}, C_{2}>0$. The existence of the constant $C_{1}$ follows from Condition 2., and that of $C_{2}$ from the compactness of $[0, \delta]$ and the analyticity of the semigroup. Noting that $n$ is asymptotic to $t / \delta$ and so $\lambda^{-n}$ is bounded by a constant times $e^{-t(\log \lambda) / \delta}$, we deduce 3 , taking $\mu=(\log \lambda) / \delta$.

3. $\Rightarrow 2 .:$ this is immediate, as indeed one can take any $\delta>0$, and then, for every $\eta \in S_{\alpha}$,

$$
\sup _{n \geq 1} e^{n \mu \delta}\left\|T\left(n \delta e^{i \alpha}+\eta\right)\right\|_{\mathcal{A}}<\infty .
$$

2. $\Rightarrow$ 4.: take $x=T(\eta)$ for $\eta \in S_{\alpha}$, and note that $x \in \Omega(\mathcal{A}) \cap\left[\bigcap_{u \in U} \mathcal{D}_{u}\right]$, where $U=\left\{\lambda^{n} T\left(n \delta e^{i \alpha}\right): n \geq 1\right\}$. With this choice we see immediately that $T\left(\delta e^{i \alpha}\right)$ is in $Q M_{r}(\mathcal{A})$.

4. $\Rightarrow 5 .:$ by the definition of $Q M_{r}(\mathcal{A})$ there exist $\lambda>0$ and $x \in \Omega(\mathcal{A})$ such that

$$
\sup _{n \geq 1}\left\|\lambda^{n} T\left(n \delta e^{i \alpha}\right) x\right\|_{\mathcal{A}}<\infty \text {. }
$$

By compactness, we have

$$
\sup _{0 \leq t \leq \delta}\left\|T\left(t e^{i \alpha}+\eta\right)\right\|_{\mathcal{A}}<\infty
$$

Hence, we get

$$
\sup _{n \geq 1,0 \leq t \leq \delta}\left\|\lambda^{n} T\left((n \delta+t) e^{i \alpha}\right) T(\eta) x\right\|_{\mathcal{A}}<\infty .
$$

Since $T(\eta) x \in \Omega(\mathcal{A})$, we obtain 5 , taking $\mu=(\log \lambda) / \delta$ as in the proof of $2 . \Rightarrow 3$. $5 . \Rightarrow 4 .:$ this is immediate.

Suppose now that there exist $\mu_{1}, \mu_{2} \in \mathbb{R}$ such that $V_{1}:=\left\{e^{-\mu_{1} t} T\left(t e^{i \alpha}\right): t>0\right\}$ and $V_{2}:=\left\{e^{-\mu_{2} t} T\left(t e^{-i \alpha}\right): t>0\right\}$ are pseudobounded. Set $V_{0}=\left\{e^{-\mu_{0}} T(t)\right\}_{t>0}$, where $\mu_{0}>\log \left(\rho(T(1))\right.$, so that $V_{0}$ is pseudobounded. Then, take $V=V_{1} \cup V_{2} \cup V_{1} V_{2}, W=V$ if $\alpha<\frac{\pi}{2}$, and set $W=V_{0} \cup V \cup V_{0} V$ if $\alpha=\frac{\pi}{2}$. Note that $V$ and $W$ are pseudobounded and stable under products. We can construct the ideal $J_{0}$ associated to $W$ as in Lemma 2.4 . Then the regular quasimultipliers $T(t)$ become multipliers on $J_{0}$ for $t \in \partial \mathcal{S}_{\alpha}$, and

$$
\left\|T\left(r e^{i \alpha}\right)\right\|_{M\left(J_{0}\right)} \leq e^{\mu_{1} r} \quad \text { and } \quad\left\|T\left(r e^{-i \alpha}\right)\right\|_{M\left(J_{0}\right)} \leq e^{\mu_{2} r}
$$

for $r>0$. Since any $t \in \mathcal{S}_{\alpha}$ can be written in the form $t=r_{1} e^{i \alpha}+r_{2} e^{-i \alpha}$, with $r_{1}>0$ and $r_{2}>0$, we see that if $\alpha<\frac{\pi}{2}$ then there exists $\nu>0$ such that $\|T(t)\|_{M\left(J_{0}\right)} \leq e^{\nu \operatorname{Re}(t)}$ for every $t \in \overline{\mathcal{S}_{\alpha}}$. Similarly if $\alpha=\frac{\pi}{2}$ we have $\|T(t)\|_{M\left(J_{0}\right)} \leq e^{\mu_{0} \operatorname{Re}(t)+\mu_{1} \operatorname{Im}(t)}$ if $\operatorname{Im}(t) \geq 0$, 
and $\|T(t)\|_{M\left(J_{0}\right)} \leq e^{\mu_{0} \operatorname{Re}(t)-\mu_{2} \operatorname{Im}(t)}$ if $\operatorname{Im}(t) \leq 0$. We obtain in particular the following result.

Proposition 3.5. Let $\alpha \in\left(0, \frac{\pi}{2}\right]$, and let $(T(t))_{t \in S_{\alpha}}$ be an analytic semigroup with unbounded generator. Assume that there exists $\delta>0$ and $\eta>0$ such that

$$
\limsup _{n \rightarrow \infty}\left\|T\left(\eta+n \delta e^{i \alpha}\right)\right\|^{\frac{1}{n}}<+\infty \quad \text { and } \quad \limsup _{n \rightarrow \infty}\left\|T\left(\eta+n \delta e^{-i \alpha}\right)\right\|^{\frac{1}{n}}<+\infty .
$$

Then there exists $\nu>0$, a commutative Banach algebra $\mathcal{B}$ and a contractive homomorphism $\theta: \mathcal{A}_{T} \rightarrow \mathcal{B}$ satisfying the following properties:

(i) $\|\theta(T(t))\| \leq e^{\nu|t|}$ for $t \in \mathcal{S}_{\alpha}$, the generator of the semigroup $\left(\theta(T(t))_{t \in \mathcal{S}_{\alpha}}\right.$ is unbounded, and there exists a strongly continuous semigroup $(\tilde{T}(t))_{t \in \overline{\mathcal{S}}_{\alpha}}$ of multipliers on $\mathcal{B}$ such that $\tilde{T}(t)=\theta(T(t))$ for $t \in \mathcal{S}_{\alpha}$.

(ii) $\theta\left(\mathcal{A}_{T}\right)$ is dense in $\mathcal{B}$, and the map $\phi: \mathcal{J} \rightarrow \overline{\theta(\mathcal{J})}^{\mathcal{B}}$ is a surjective map from the set of closed ideals of $\mathcal{A}_{T}$ onto the set of closed ideals of $\mathcal{B}$. If, further, $\lim \sup _{t \rightarrow 0^{+}}\|T(t)\|<$ $+\infty$, then $\phi$ is a bijection.

Proof. We can assume without loss of generality that $\mathcal{A}_{T}^{\perp}=\{0\}$, since otherwise we can construct $\nu>0$, a commutative Banach algebra $\mathcal{B}$ and a map $\theta: \mathcal{A}_{T} / \mathcal{A}_{T}^{\perp} \rightarrow \mathcal{B}$ with the required properties, and $\theta \circ \pi: \mathcal{A}_{T} \rightarrow \mathcal{B}$ satisfies the conditions of the proposition, where $\pi: \mathcal{A}_{T} \rightarrow \mathcal{A}_{T} / \mathcal{A}_{T}^{\perp}$ denotes the canonical surjection. It follows from the fact that 4 implies 5. in Proposition 3.4 that there exists $\mu_{1}, \mu_{2} \in \mathbb{R}$ such that $V_{1}:=\left\{e^{-\mu_{1} t} T\left(t e^{i \alpha}\right): t>0\right\}$ and $V_{2}:=\left\{e^{-\mu_{2} t} T\left(t e^{-i \alpha}\right): t>0\right\}$ are pseudobounded. We can define $V$ and $W$ as above and construct the ideal $J_{0}$ associated to $W$ as in Lemma 2.4. Then the regular quasimultipliers $T(t)$ become multipliers on $J_{0}$ for $t \in \partial \mathcal{S}_{\alpha}$, which gives a semigroup $\tilde{T}(t)$ on $\overline{\mathcal{S}_{\alpha}}$ in $M\left(J_{0}\right)$ which extends $(\theta(t))_{t \in \mathcal{S}_{\alpha}}$, where we denote by $\theta: \mathcal{A} \rightarrow \mathcal{B}$ the natural injection. Denote by $\mathcal{B}$ the closure of $\theta\left(\mathcal{A}_{T}\right)$ (or, equivalently, the closure of $J_{0}$ ) in $\left(M\left(J_{0}\right),\|\cdot\|_{M\left(J_{0}\right)}\right)$. It follows from the definition of $J_{0}$ that $R(x) \in J_{0}$ for every $x \in J_{0}$ and every $R \in M(\mathcal{B})$, and so $M\left(J_{0}\right)=M(\mathcal{B})$.

The algebras $\mathcal{A}_{T}$ and $\mathcal{B}$ are similar in the sense of [9], which obviously implies that $\mathcal{B}$ is nonunital since $\mathcal{A}_{T}$ is nonunital. Hence the generator of the semigroup $\left(\theta(T(t))_{t \in \mathcal{S}_{\alpha}}\right.$ is unbounded. It follows from the discussion above that there exists $\mu>0$ such that $\|\theta(T(t))\| \leq e^{\mu|t|}$ for $t \in \mathcal{S}_{\alpha}$. Since the algebra generated by $(\theta(t))_{t \in \mathcal{S}_{\alpha}}$ is dense in $\mathcal{B}$, the semigroup $(\tilde{T}(t))_{t \in \overline{\mathcal{S}_{\alpha}}}$ is strongly continuous. Assertion (ii) follows from an elementary argument given in the proof of Proposition 7.3 (2), p. 113, and Proposition 7.8 (2), p. 122, in 9 .

In the general case of an analytic semigroup in a sector, a similar result remains true with a weaker conclusion.

Proposition 3.6. Let $\alpha \in\left(0, \frac{\pi}{2}\right]$, and let $(T(t))_{t \in S_{\alpha}}$ be an analytic semigroup with unbounded generator. Then there exists $\nu>0$, a commutative Banach algebra $\mathcal{B}$ and a contractive homomorphism $\theta: \mathcal{A}_{T} \rightarrow \mathcal{B}$ satisfying the following properties:

(i) For every $\beta \in(0, \alpha)$ there exists $\nu_{\beta}>0$ such that $\|\theta(T(t))\| \leq e^{\nu_{\beta} \operatorname{Re}(t)}$ for every $t \in \overline{\mathcal{S}_{\beta}}$, and the generator of the semigroup $\left(\theta(T(t))_{t \in \mathcal{S}_{\alpha}}\right.$ is unbounded. 
(ii) $\theta\left(\mathcal{A}_{T}\right)$ is dense in $\mathcal{B}$, and the map $\phi: \mathcal{J} \rightarrow \overline{\theta(\mathcal{J})}^{\mathcal{B}}$ is a surjective map from the set of closed ideals of $\mathcal{A}_{T}$ onto the set of closed ideals of $\mathcal{B}$. If, further, $\lim _{\sup _{t \rightarrow 0^{+}}}\|T(t)\|<$ $+\infty$, then $\phi$ is a bijection.

Proof. Since there exists $\mu_{0}>0$ such that the set $W:=\left\{e^{-\mu_{0} t} T(t)\right\}_{t>0}$ is pseudobounded, we can use the same procedure as in Proposition 3.5, which allows us to assume without loss of generality that $\|T(t)\| \leq e^{\mu_{0} t}$ for $t>0$. Set $\beta_{n}=\frac{\alpha n}{n+1}$ for $n \geq 1$.

We can define by induction a sequence $\left(\mu_{n}\right)_{n \geq 1}$ of positive real numbers such that we have

$$
\begin{aligned}
& \sup _{r>0} e^{-\mu_{n} r}\left\|T\left(2^{-n}\right) T\left(r e^{i \beta_{n}}\right)\right\|<e^{2^{-n} \mu_{0}}\left(1+2^{-n}\right), \\
& \sup _{r>0} e^{-\mu_{n} r}\left\|T\left(2^{-n}\right) T\left(r e^{-i \beta_{n}}\right)\right\|<e^{2^{-n} \mu_{0}}\left(1+2^{-n}\right) .
\end{aligned}
$$

To see this, notice that $\lim _{r \rightarrow+\infty} e^{-\mu r}\left\|T\left(2^{-n}\right) T\left(r e^{ \pm i \beta_{n}}\right)\right\|=0$ when $\mu$ is sufficiently large. This shows that for every $\delta>0$ there exists $\sigma_{\delta}>0$ such that

$$
e^{-\sigma_{\delta} r}\left\|T\left(2^{-n}\right) T\left(r e^{ \pm i \beta_{n}}\right)\right\|<e^{2^{-n} \mu_{0}}\left(1+2^{-n}\right)
$$

for $r>\delta$. So the two conditions are satisfied, taking $\mu_{n}=\frac{\sigma_{\delta}}{\cos \left(\beta_{n}\right)}$ when $\delta$ is sufficiently small. Set

$$
V_{1, n}=\left\{e^{-\mu_{n} r} T\left(2^{-n}\right) T\left(r e^{i \beta_{n}}\right)\right\}_{r>0} \cup\{I\}, \quad V_{2, n}=\left\{e^{-\mu_{n} r} T\left(2^{-n}\right) T\left(r e^{-i \beta_{n}}\right)\right\}_{r>0} \cup\{I\},
$$

and

$$
W=\bigcup_{n \geq 1} V_{1,1} \ldots V_{1, n} V_{2,1} \ldots V_{2, n} .
$$

Then $W$ is stable under products and pseudobounded, since $\sup _{w \in W}\|T(2) w\|<+\infty$. Applying to $W$ the same procedure as in the proof of Proposition 3.5, we obtain the result.

REMARK 3.7. Assume that $\mathcal{A}^{\perp}=\{0\}$. If the generator of the semigroup is unbounded, we have $\lim \sup _{n \rightarrow \infty}\|T(-n \delta) x\|^{\frac{1}{n}}=+\infty$ for every nonzero $x \in \bigcap_{t>0} T(t) \mathcal{A}_{T} \cap \Omega(A)$, and $\lim \sup _{n \rightarrow+\infty}\left\|\Delta^{n} x\right\|^{\frac{1}{n}}=+\infty$ for every nonzero $x \in \bigcap_{n \geq 1} \operatorname{Dom}\left(\Delta^{n}\right) \cap \Omega\left(\mathcal{A}_{T}\right)$.

Proof. Assume that

$$
\limsup _{n \rightarrow \infty}\|T(-n \delta) x\|^{\frac{1}{n}}<+\infty
$$

for some $x \in \Omega(A) \cap \bigcap_{t>0} T(t) \mathcal{A}_{T}$. Then $T(-\delta)$ would be a regular quasimultiplier on $\mathcal{A}_{T}$. Applying the procedure of Lemma 2.4 we would obtain a commutative Banach algebra $\mathcal{B}$ containing $\mathcal{A}_{T}$ and an ideal $J_{0}$ of $\mathcal{A}_{T}$ which is also a dense ideal of $\mathcal{B}$ such that $T(-\delta)$ is a bounded multiplier on $\mathcal{B}$. This would imply that $\lim _{t \rightarrow 0^{+}}\|I-T(t)\|=0$, and $\mathcal{B}$ would be unital. Hence $J_{0}=\mathcal{B}, \mathcal{A}_{T}=\mathcal{B}$, and $\mathcal{A}_{T}$ would be unital, which is impossible. A similar argument shows that if the generator of an analytic semigroup is unbounded, then it is not a regular quasimultiplier.

Recall that a semigroup is quasinilpotent if $\rho(T(t))$, the spectral radius of $T(t)$, equals zero for each $t>0$ (or, in the case of a sectorial semigroup, for each $t \in S_{\alpha}$ ). This coincides with the notion of superstability studied by Balakrishnan [2]. An example of a 
quasinilpotent analytic semigroup on $L^{p}(0,1)$ for $p \geq 1$ was given in [11, pp. 663-664]. It is defined by

$$
T(t) f(x)=\frac{1}{\Gamma(t)} \int_{0}^{x}(x-u)^{t-1} f(u) d u \quad(\operatorname{Re} t>0) .
$$

For quasinilpotent semigroups, we have in fact $\liminf _{n \rightarrow \infty}\|T(-n \delta) x\|^{\frac{1}{n}}=+\infty$ for every nonzero $x \in \bigcap_{t>0} T(t) \mathcal{A}_{T}$, and $\liminf _{n \rightarrow+\infty}\left\|\Delta^{n} x\right\|^{\frac{1}{n}}=+\infty$ for every nonzero $x \in \bigcap_{p \geq 1} \operatorname{Dom}\left(\Delta^{p}\right)$.

To see this assume that $S$ is a quasinilpotent bounded operator on a Banach space $\mathcal{X}$, that $x \in \cap_{n \geq 1} S^{n}(\mathcal{X})$, and that a sequence $\left(x_{n}\right)_{n \geq 1}$ of elements of $\mathcal{X}$ satisfies $S^{n} x_{n}=x$. If $x \neq 0$, we have

$$
\left\|x_{n}\right\|^{\frac{1}{n}} \geq \frac{\|x\|^{\frac{1}{n}}}{\left\|S^{n}\right\|^{\frac{1}{n}}}
$$

and so $\liminf \inf _{n \rightarrow+\infty}\left\|x_{n}\right\|^{\frac{1}{n}}=+\infty$.

Now if a quasinilpotent strongly continuous semigroup $(T(t))$ of bounded operators on a Banach space $\mathcal{X}$ is bounded near the origin, we can define a bounded operator on $X$ by the formula

$$
R=\int_{0}^{+\infty} T(t) d t
$$

where the Bochner integral is computed with respect to the strong operator topology; then $R \Delta x=x$ for every $x \in \operatorname{Dom}(\Delta)$, where $\Delta$ denotes the generator of the semigroup. Similarly the formula

$$
\phi(f)=\int_{0}^{+\infty} f(t) T(t) d t
$$

defines a homomorphism from the convolution algebra $\bigcup_{a>0} L^{1}\left(\mathbb{R}^{+}, e^{a t} d t\right)$ into $\mathcal{B}(\mathcal{X})$, and $R=\phi(1)$. Hence we have

$$
R^{n}=\phi\left(1^{* n}\right)=\int_{0}^{+\infty} \frac{t^{n-1}}{(n-1) !} T(t) d t,
$$

and $R^{n} \Delta^{n} x=x$ for every $x \in \operatorname{Dom}\left(\Delta^{n}\right)$.

If the semigroup is not bounded near the origin, we can nevertheless define for $\alpha>0$ and $n \geq 1$ a bounded operator $R_{\alpha, n}$ by using the formula

$$
R_{\alpha, n}=\int_{0}^{+\infty} \frac{t^{n-1}}{(n-1) !} T(\alpha+t) d t
$$

and $R_{\alpha, n} \Delta^{n} x=T(\alpha) x$ for every $x \in \operatorname{Dom}\left(\Delta^{n}\right)$.

For every $p \geq 1$, there exists $M_{p, \alpha}>0$ such that we have, for every $n \geq 1$,

$$
\left\|R_{\alpha, n}\right\| \leq M_{\alpha, p} \int_{0}^{+\infty} \frac{t^{n-1}}{(n-1) !} e^{-t p} d t=\frac{1}{p^{n}},
$$

and so $\lim _{n \rightarrow+\infty}\left\|R_{\alpha, n}\right\|^{\frac{1}{n}}=0$. Since $T(\alpha) x \neq 0$ when $\alpha$ is sufficiently small for every $x \in \operatorname{Dom}(\Delta)$, we obtain, for every nonzero $x \in \bigcap_{n \geq 1} \operatorname{Dom}\left(\Delta^{n}\right)$,

$$
\liminf _{n \rightarrow+\infty}\left\|\Delta^{n} x\right\|^{\frac{1}{n}}=+\infty .
$$


4. Inequalities for analytic semigroups. The next lemma demonstrates that nontrivial quasinilpotent analytic semigroups cannot be bounded on the right half-plane. In fact, more is true.

LEMMA 4.1. Let $(T(t))_{\operatorname{Re} t>0}$ be a quasinilpotent analytic semigroup of bounded operators on a Banach space $\mathcal{X}$. Suppose that there exists $r>0$ such that

$$
\sup _{t \in D^{+}(0, r)}\|T(t)\|<+\infty
$$

and define $T($ iy) for $y \in \mathbb{R}$ by Remark 3.2. If

$$
\int_{-\infty}^{\infty} \frac{\log ^{+}\|T(i y)\|}{1+y^{2}} d y<+\infty
$$

then $T(t)=0$ for $\operatorname{Re} t>0$.

Proof. Take a nonzero $x \in \mathcal{X}$ and $\phi \in \mathcal{X}^{*}$, and define $f$ by $f(t)=\phi(T(t) x)$. Since each $T(t)$ is quasinilpotent, it follows that

$$
\frac{\log \left|f\left(r e^{i \theta}\right)\right|}{r} \rightarrow-\infty
$$

as $r$ tends to $+\infty$ and for all $\theta \in(-\pi / 2, \pi / 2)$. Now, recall that the Ahlfors-Heins theorem (see [7, Thm A.2.47] or [6, Thm 7.1]) asserts that if $g$ is a not identically zero function of exponential type in $\mathbb{C}^{+}:=\{z \in \mathbb{C}: \operatorname{Re}(z)>0\}$, such that

$$
\int_{-\infty}^{\infty} \frac{\log ^{+}|g(i y)|}{1+y^{2}} d y<\infty
$$

then

$$
c=\lim _{r \rightarrow \infty} \frac{2}{\pi r} \int_{-\pi / 2}^{\pi / 2} \log \left|g\left(r e^{i \theta}\right)\right| \cos \theta d \theta
$$

exists in $\mathbb{R}$, and for almost all $\theta \in(-\pi / 2, \pi / 2)$,

$$
\lim _{r \rightarrow \infty} \frac{\log \left|g\left(r e^{i \theta}\right)\right|}{r}=c \cos \theta .
$$

It follows that $f$ is identically equal to 0 . Then by the Hahn-Banach theorem, since the above conclusion holds for any $x \in \mathcal{X}$ and $\phi \in \mathcal{X}^{*}$, we have that $T(t)$ is identically equal to 0 .

We are now ready to make estimates for semigroups on the imaginary axis.

THEOREM 4.2. Let $(T(t))_{\operatorname{Re} t>0}$ be a nonzero quasinilpotent analytic semigroup satisfying the conditions of Remark 3.2, and let $s>0$. Then

$$
\max (\rho(T(i y)-T(i y+i s)), \rho(T(-i y)-T(-i y-i s))) \geq 2,
$$

for every $y>0$.

Proof. Assume that $\lambda \neq-|\lambda|$ for every $\lambda$ in $\operatorname{Spec}(T(i s))$, the spectrum of $T(i s)$. Then there exists a determination of $\log (z)$ which is analytic on a neighbourhood of $\operatorname{Spec}(T(i s))$, which means that there exists a bounded operator $U$ on $\mathcal{X}$ such that $T(i s)=e^{U}$. Now set $\tilde{T}(t)=T(t) e^{i t U / s}$. Then $\tilde{T}(t)$ is quasinilpotent for $\operatorname{Re} t>0$, and $\tilde{T}(i s)=\operatorname{Id}=\tilde{T}(0)$. 
So $\tilde{T}(i y)$ is periodic, hence bounded for $y \in \mathbb{R}$. Using Lemma 4.1, $T(t)=\tilde{T}(t)=0$ for Re $t \geq 0$, a contradiction.

Thus we may take $\lambda \in \operatorname{Spec}(T(i s))$ such that $\lambda=-|\lambda|$. Hence

$$
\max (\rho(\operatorname{Id}-T(i s)), \rho(\operatorname{Id}-T(-i s))) \geq \max \left(1+|\lambda|, 1+|\lambda|^{-1}\right) \geq 2 .
$$

Let $\mathcal{B}$ be a maximal commutative subalgebra of $\mathcal{L}(\mathcal{X})$ containing $T(t)$ for $\operatorname{Re} t \geq 0$. There exists a character $\chi$ on $\mathcal{B}$ such that $\chi(T(i s))=\lambda$. We have, for $y \in \mathbb{R}$,

$$
|\chi(T(i y)-T(i y+i s))|=|\chi(T(i y))||1-\chi(T(i s))|=|\chi(T(i y))|(1+|\lambda|),
$$

and

$$
|\chi(T(-i y)-T(-i y-i s))|=|\chi(T(-i y))||1-\chi(T(-i s))|=\frac{1}{|\chi(T(i y))|}\left(1+\frac{1}{|\lambda|}\right) .
$$

Since the group $(T(i y))_{y \in \mathbb{R}}$ is strongly continuous, it follows from the Banach-Steinhaus theorem that this group is locally bounded, which implies that there exists $\alpha \in \mathbb{R}$ such that $|\chi(T(i y))|=e^{\alpha y}$ for $y \in \mathbb{R}$. Now let $y>0$. If $|\lambda| \geq 1$, then $\alpha \geq 0,|\chi(T(i y))| \geq 1$, and so

$$
|\chi(T(i y)-T(i y+i s))| \geq 2 .
$$

If $|\lambda|<1$, then $\alpha<0,|\chi(T(i y))|<1$, and so

$$
|\chi(T(-i y)-T(-i y-i s))|>2 .
$$

Combining these, it follows that

$$
\max (|\chi(T(i y)-T(i y+i s))|,|\chi(T(-i y)-T(-i y-i s))|) \geq 2,
$$

which proves the theorem.

COROLlary 4.3. Let $(T(t))_{\operatorname{Re} t>0}$ be a quasinilpotent analytic semigroup such that

$$
\sup _{y \in \mathbb{R}} e^{-\mu|y|}\|T(\delta+i y)\|<+\infty
$$

for some $\delta>0$ and some $\mu>0$, and let $\gamma>0$. Then

$$
\sup _{t \in D^{+}(0, r)}\|T(t)-T((1+\gamma) t)\| \geq 2
$$

for every $r>0$.

Proof. It follows from Proposition 3.5 that we may assume without loss of generality that $\sup _{z \in D^{+}(0, \rho)}\|T(t)\|<+\infty$ for some $\rho>0$. Since $T(i y)-T(i(1+\gamma) y)$ is the limit of $T(i y+\varepsilon)-T((1+\gamma)(i y+\varepsilon))$ as $\varepsilon \rightarrow 0^{+}$with respect to the strong operator topology, we have, for every $y \in \mathbb{R}$,

$$
\begin{aligned}
\rho(T(i y)-T((1+\gamma) i y)) & \leq\|T(i y)-T((1+\gamma) i y)\| \\
& \leq \liminf _{\varepsilon \rightarrow 0^{+}}\|T(i y+\varepsilon)-T((1+\gamma)(i y+\varepsilon))\| .
\end{aligned}
$$

Hence, by Theorem 4.2

$$
\max _{\eta \in\{-1,1\}} \liminf _{\varepsilon \rightarrow 0^{+}}\|T(i \eta y+\varepsilon)-T((1+\gamma)(i \eta y+\varepsilon))\| \geq 2
$$

for every $y>0$, and the corollary follows. 
Acknowledgements. This paper is based on a lecture delivered at the $19^{\text {th }}$ International Conference on Banach Algebras held at Będlewo, July 14-24, 2009. The support for the meeting by the Polish Academy of Sciences, the European Science Foundation under the ESF-EMS-ERCOM partnership, and the Faculty of Mathematics and Computer Science of the Adam Mickiewicz University at Poznań is gratefully acknowledged. The first and third authors are also grateful for financial support from the British EPSRC; the second author likewise thanks the French ANR project AHPI.

\section{References}

[1] G. R. Allan, H. G. Dales, and J. P. McClure, Pseudo-Banach algebras, Studia Math. 40 (1971), 55-69.

[2] A. V. Balakrishnan, On superstability of semigroups, in: Systems Modelling and Optimization (Detroit, MI, 1997), Chapman \& Hall/CRC Res. Notes Math. 396, Chapman \& Hall/CRC, Boca Raton, FL, 1999, 12-19.

[3] Z. Bendaoud, I. Chalendar, J. Esterle, and J. R. Partington, Distances between elements of a semigroup and estimates for derivatives, Acta Mathematica Sinica 26 (2010), 2239-2254.

[4] A. Beurling, On analytic extension of semigroups of operators, J. Funct. Anal. 6 (1970), $387-400$.

[5] R. P. Boas, Jr, Entire Functions, Academic Press, New York, 1954.

[6] H. G. Dales, Convolution algebras on the real line, in: Radical Banach Algebras and Automatic Continuity (Long Beach, Calif., 1981), Lecture Notes in Math. 975, Springer, Berlin, 1983, 180-209.

[7] H. G. Dales, Banach Algebras and Automatic Continuity, London Mathematical Society Monographs, New Series 24, Oxford University Press, New York, 2000.

[8] J. Esterle, Elements for a classification of commutative radical Banach algebras, in: Radical Banach Algebras and Automatic Continuity (Long Beach, Calif., 1981), Lecture Notes in Math. 975, Springer, Berlin, 1983, 4-65.

[9] J. Esterle, Quasimultipliers, representations of $H^{\infty}$, and the closed ideal problem for commutative Banach algebras, in: Radical Banach Algebras and Automatic Continuity (Long Beach, Calif., 1981), Lecture Notes in Math. 975, Springer, Berlin, 1983, 66-162.

[10] W. Feller, On the generation of unbounded semi-groups of bounded linear operators, Ann. of Math. (2) 58 (1953), 166-174.

[11] E. Hille and R. S. Phillips, Functional Analysis and Semi-Groups, rev. ed. American Mathematical Society Colloquium Publications 31, American Mathematical Society, Providence, R.I., 1957.

[12] L. Hörmander, An Introduction to Complex Analysis in Several Variables, North-Holland Mathematical Library 7, North-Holland, Amsterdam, third edition, 1990.

[13] T. Kato, A characterization of holomorphic semigroups, Proc. Amer. Math. Soc. 25 (1970), 495-498. 
